\title{
Notch signaling suppresses glucose metabolism in mesenchymal progenitors to restrict osteoblast differentiation
}

\author{
Seung-Yon Lee ${ }^{1}$ and Fanxin Long ${ }^{1,2}$ \\ 'Department of Orthopaedic Surgery, and 2Department of Developmental Biology, Washington University School of Medicine, St. Louis, Missouri, USA.
}

\begin{abstract}
Notch signaling critically controls cell fate decisions in mammals, both during embryogenesis and in adults. In the skeleton, Notch suppresses osteoblast differentiation and sustains bone marrow mesenchymal progenitors during postnatal life. Stabilizing mutations of Notch2 cause Hajdu-Cheney syndrome, which is characterized by early-onset osteoporosis in humans, but the mechanism whereby Notch inhibits bone accretion is not fully understood. Here, we report that activation of Notch signaling by either Jagged1 or the Notch2 intracellular domain suppresses glucose metabolism and osteoblast differentiation in primary cultures of bone marrow mesenchymal progenitors. Importantly, deletion of Notch2 in the limb mesenchyme increases both glycolysis and bone formation in the long bones of postnatal mice, whereas pharmacological reduction of glycolysis abrogates excessive bone formation. Mechanistically, Notch reduces the expression of glycolytic and mitochondrial complex I genes, resulting in a decrease in mitochondrial respiration, superoxide production, and AMPK activity. Forced activation of AMPK restores glycolysis in the face of Notch signaling. Thus, suppression of glucose metabolism contributes to the mechanism, whereby Notch restricts osteoblastogenesis from bone marrow mesenchymal progenitors.
\end{abstract}

\section{Introduction}

Notch signaling between neighboring cells critically controls cell fate decisions in all metazoans $(1,2)$. In mammalian cells, engagement of the Notch receptors (Notch1-4) by the ligands (Jagged1 and -2 and Delta-like 1, -3 , and -4) triggers sequential proteolysis of the receptors, which culminates in intramembranous cleavages by the $\gamma$-secretase complex and results in release of the Notch intracellular domain (NICD) (3-5). In the best-studied "canonical" mechanism, NICD released from the plasma membrane translocates to the nucleus, where it interacts with the transcription factor RBPjk and a mastermind-like transcriptional coactivator (Maml1-3) to activate the transcription of target genes (6). Among the best known targets of Notch signaling are the Hes/ Hey family of basic helix-loop-helix (bHLH) transcription repressors (7). However, the regulation of individual Hes/Hey proteins by Notch and their role in mediating Notch function are highly dependent on cell context.

Mouse genetic studies have uncovered important functions for Notch signaling in bone. Genetic deletion of either the $\gamma$-secretase catalytic subunits presenilin 1 and -2 , or Notch 1 and -2 in the embryonic limb mesenchyme results in excessive osteoblast differentiation at the expense of bone marrow mesenchymal progenitors (8). More recently, specific deletion of Notch2 in early-stage osteoblast lineage cells with Runx2-Cre increased trabecular bone

Conflict of interest: The authors have declared that no conflict of interest exists. License: Copyright 2018, American Society for Clinical Investigation.

Submitted: July 11, 2017; Accepted: October 2, 2018.

Reference information: J Clin Invest. 2018;128(12):5573-5586.

https://doi.org/10.1172/JCI96221 mass in a site-specific manner (9). In addition, Jagged1 appears to be a major ligand in the maintenance of the osteoprogenitor pool (10). Consistent with the negative role of Notch in osteoblast differentiation, forced expression of NICD in osteoblastic precursors reduced osteoblast numbers and caused osteopenia (11). Intriguingly, forced expression of NICD at a later stage of the osteoblast lineage led to sclerosis owing to excessive proliferation of the immature osteoblasts, highlighting stage-specific functions of constitutive Notch activation in the osteoblast lineage (12). Abnormal Notch activation in mesenchymal progenitors has also been implicated in decreased osteoblast differentiation associated with rheumatoid arthritis (13). In keeping with the mouse studies, Notch1 haploinsufficiency in humans causes ectopic osteoblast differentiation and calcification in the aortic valves $(14,15)$, whereas Notch2 stabilizing mutations are responsible for Hadju-Cheney syndrome (HCS), a disorder of severe and progressive bone loss $(16,17)$. Mice carrying an HCS mutation knockin allele through the germ line developed severe osteopenia due to increased bone resorption (18). More recently, a mouse strain expressing a conditional Notch2-knockin allele lacking the PEST degradation signal was created, and activation of the allele specifically in osteoblasts resulted in osteopenia due to increased Rankl expression, which promotes osteoclastogenesis (19). In all cases, however, the mechanisms through which Notch signaling inhibits osteoblast differentiation from the progenitors have not been fully elucidated.

Recent studies have implicated the rewiring of cellular metabolism in osteoblast differentiation. In particular, Wnt/ Lrp5 signaling, which promotes bone formation in both mice and humans, has been shown to stimulate aerobic glycolysis and glutamine catabolism as well as fatty acid oxidation during 
A
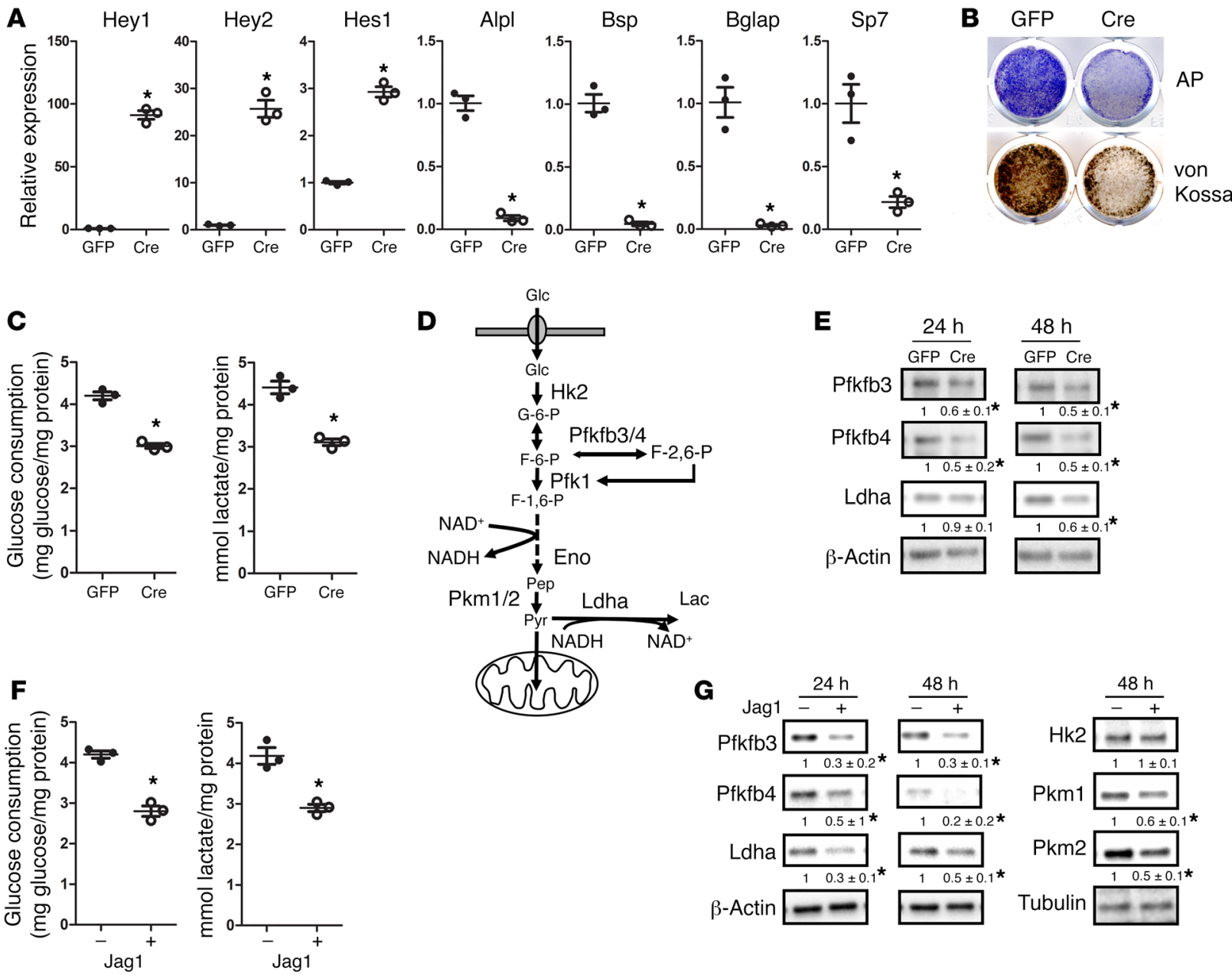

Figure 1. Notch signaling suppresses glycolysis in bone marrow mesenchymal progenitors. (A) Relative mRNA levels of the indicated genes assayed by RT-qPCR in R26-NICD2 BMSCs infected with Ad-GFP or Ad-Cre and treated with mineralization media for 48 hours. $n=3$. (B) AP or von Kossa staining in R26-NICD2 BMSCs infected with Ad-GFP or Ad-Cre after 4 days or 2 weeks, respectively, in mineralization media. (C) Glucose consumption and lactate production by R26-NICD2 BMSCs infected with Ad-GFP or Ad-CRE in regular growth media for 48 hours. $n=3$. (D) Diagram of glycolysis and its key enzymes. (E) Western blots in R26-NICD2 BMSCs infected with Ad-GFP or Ad-CRE for 24 or 48 hours. Protein levels were normalized to $\beta$-actin and designated 1 in samples infected with Ad-GFP. Quantification (mean \pm SD) was determined from 3 independent samples. (F) Glucose consumption and lactate production from WT BMSCs with or without Jagged1 stimulation for 48 hours. $n=3$. (C) Western blots in WT BMSCs with or without Jagged1 stimulation for the indicated durations. Protein levels were normalized to $\beta$-actin or tubulin, and quantification (mean \pm SD) was determined from 3 independent samples. ${ }^{*} P<0.05$, by 2-tailed Student's $t$ test (A, C, and E-C). Hk2: hexokinase 2; Pfkfb3/4: 6-phosphofructo-2-kinase/fructose-2,6-bisphosphatase 3 or 4; F-2,6-P: fructose 2,6-bisphosphate; Pfk1: phosphofructokinase 1; Eno: enolase; Ldha: lactate dehydrogenase a; Pkm: pyruvate kinase, muscle.

osteoblastogenesis (20-22). In addition, teriparatide, a fragment (amino acids 1-34) of human parathyroid hormone (PTH) and an FDA-approved bone anabolic drug, enhances aerobic glycolysis via the induction of insulin-like factor (Igf) signaling (23). Runx2, a critical transcription factor for osteoblast differentiation, appears to engage Glut1, the major glucose transporter in osteogenic cells, in a feed-forward regulation to control osteoblast differentiation and bone formation in the mouse (24). Interestingly, Notch signaling has been shown to affect cellular metabolism in other systems. Both hyper- and hypoactivation of Notch induced a glycolytic phenotype in breast cancer cells through distinct mechanisms (25). Forced expression of NICD1 in a cell culture model altered the mitochondrial proteome involved in oxidative phosphorylation, glutamine catabolism, and fatty acid oxidation (26). In Drosophila cells, Notch stimulated glycolysis while sup- pressing metabolism through the TCA cycle (27). However, in the mouse, Notch signaling has been shown to suppress glycolysis in endothelial cells (28). Notch activation was also linked with the increase in both glycolysis and mitochondrial oxidative phosphorylation in murine proinflammatory macrophages (29). Thus, it is likely that Notch controls cellular metabolism in a contextdependent manner. A potential role for the metabolic regulation of Notch in bone has yet to be explored.

Here, we report that Notch signaling diminished glycolysis along with suppression of osteoblast differentiation in bone marrow mesenchymal progenitors. Deletion of Notch2 concurrently increased glycolysis and osteoblast numbers in the mouse. Pharmacological reduction of the glycolytic flux abrogated excessive bone formation in Notch2-deficient mice. Thus, Notch signaling impedes bone formation in part through suppression of glucose metabolism. 
A

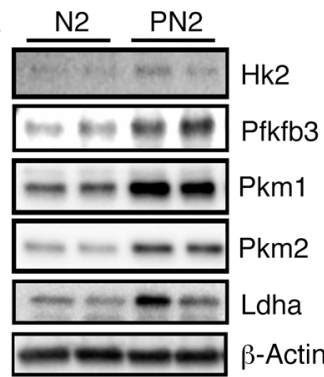

B

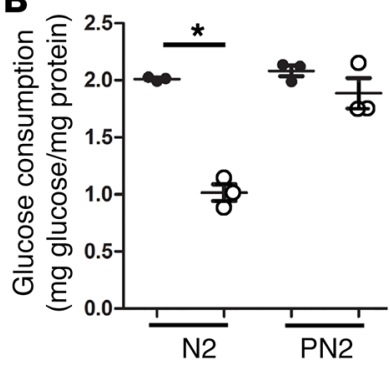

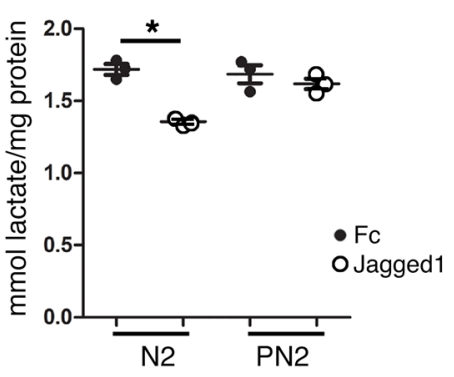

$\mathbf{F}$

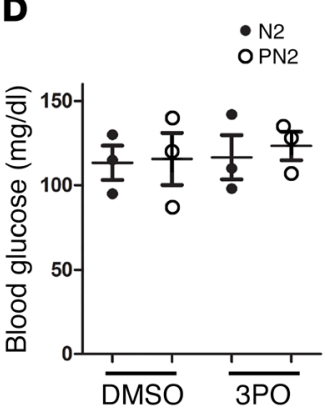

$\mathbf{E}$

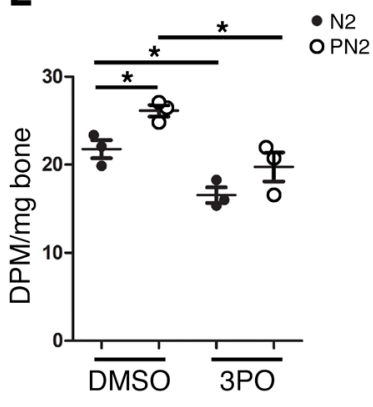

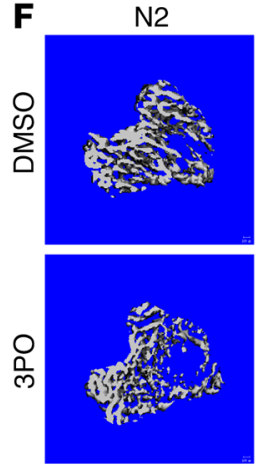

PN2

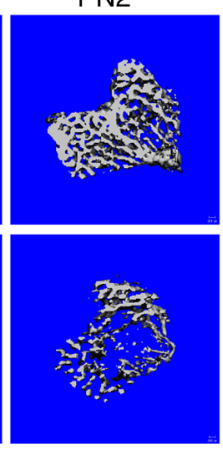

C

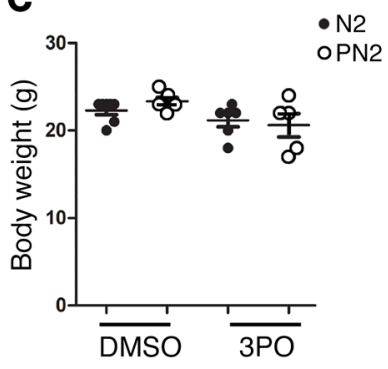

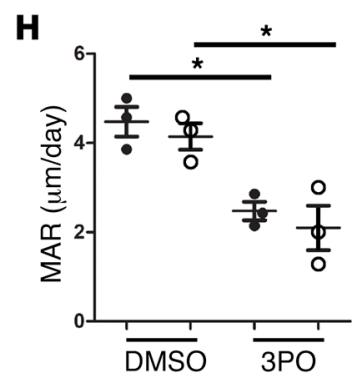
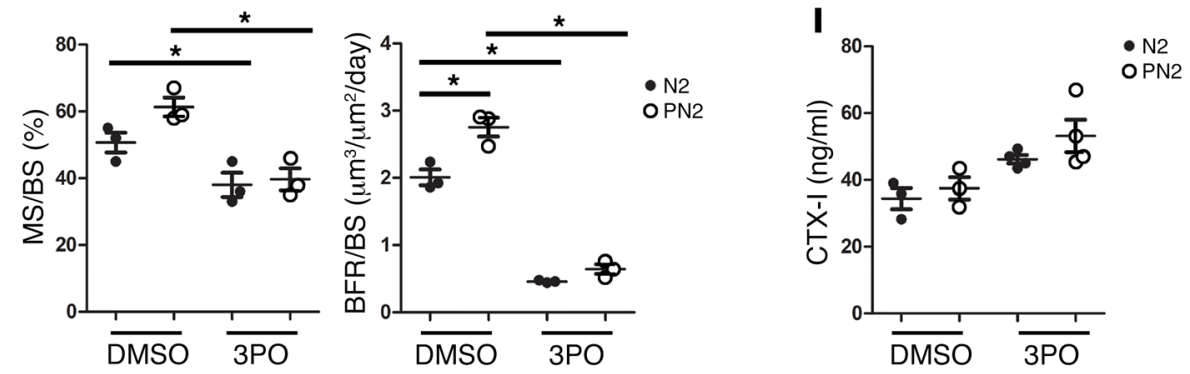

Figure 2. Suppression of glycolysis abolishes excessive bone formation caused by Notch2 deletion. (A) Western blots of long bones from 8-week-old Prx1-Cre Notch2 ${ }^{\mathrm{fl} / \mathrm{fl}}$ (PN2) mice and Notch2 ${ }^{\mathrm{fl} / \mathrm{fl}}$ (N2) littermates. Each lane is for a separate mouse. (B) Glucose consumption and lactate production in N2 and PN2 BMSCs with or without Jagged1 treatment for 48 hours. $n=3$. (C-I) Analyses of 7-week-old N2 or PN2 mice that received 3 PO or DMSO for 4 weeks starting at 3 weeks of age, including body weight (C), blood glucose (D), ${ }^{14} C-2 D C$ uptake in bone (E), $\mu C T$ images (F), $\mu$ CT quantification (C), and dynamic histomorphometry $(\mathbf{H})$ of trabecular bone in the proximal tibia, as well as serum CTX-I levels $(\mathbf{I}) . n=3 .{ }^{*} P<0.05$, by 2 -way ANOVA followed by Bonferroni's post hoc test (C-E, H, and I) or 2-tailed Student's $t$ test (B and $\mathbf{G})$. BV, bone volume; DPM, disintegrations per minute; TV, total volume.

\section{Results}

Notch signaling suppresses glycolysis in bone marrow mesenchymal progenitors. HCS is caused by abnormal stabilization of the Notch2 ICD (NICD2) following activation of the Notch2 receptor. The increased half-life of NICD2 probably leads to elevated NICD2 levels and a prolonged Notch response in the cell. To model this aspect of HCS, we overexpressed NICD2 in primary cultures of bone marrow stromal cells (BMSCs). Specifically, we used the R26-NICD2 mouse, which was genetically engineered to express NICD2 from the ubiquitously active Rosa26 locus upon Cre recombination (30). Briefly, BMSCs were cultured from young adult mice harboring the R26-NICD2 allele and infected with adenovirus expressing either Cre (Ad-Cre) or GFP as a control (Ad-GFP). As BMSCs contain mesenchymal progenitors with the potential to become osteoblasts, we assessed the effect of NICD2 overexpression on osteoblastogenesis in response to ascorbic acid and $\beta$-glycerophosphate. After 48 hours of treatment, NICD2 induced the Notch target genes
Hey1, Hey2, and Hes1, but markedly suppressed expression of the osteoblast markers Alpl, Bsp, Bglap, and Sp7 in BMSCs (Figure 1A). After 4 days or 2 weeks of differentiation, the Ad-Cre-infected cells showed less alkaline phosphatase (AP) or von Kossa staining, respectively, than did the control cells (Figure 1B). Thus, sustained Notch2 signaling suppresses osteoblast differentiation from bone marrow mesenchymal progenitors.

In light of recent evidence implicating glucose metabolism in osteoblast differentiation, we investigated the effect of Notch2 activation on glycolysis. When BMSCs from R26-NICD2 mice were infected with Ad-Cre or Ad-GFP, those infected with Ad-Cre consumed less glucose and secreted less lactate after 48 hours (Figure 1C). To assess the biochemical basis for the glycolytic suppression, we examined the abundance of several enzymes controlling key steps of glycolysis (Figure 1D). Among those, Pfk$\mathrm{fb} 3$ and Pfkfb4 increase the amount of fructose-2,6-bisphosphate (F-2,6-P) that stimulates the activity of phosphofructokinase Pfk1, 
Table 1. $\mu \mathrm{CT}$ analyses of trabecular bone in proximal tibiae

\begin{tabular}{|c|c|c|c|c|}
\hline Genotype & BV/TV (\%) & Tb.N (1/mm) & Tb.Th (mm) & Tb.Sp (mm) \\
\hline Notch $2^{f \mid / 1 / 1} / D M S O$ & $18.1 \pm 1.108$ & $4.16 \pm 0.335$ & $0.0679 \pm 0.002$ & $0.240 \pm 0.025$ \\
\hline Notch $2^{\mathrm{f} / \mathrm{fl}} / 3 \mathrm{PO}$ & $12.1 \pm 2.661^{A}$ & $2.81 \pm 0.537^{A}$ & $0.0613 \pm 0.006^{A}$ & $0.365 \pm 0.079^{A}$ \\
\hline Prx1-Cre Notch2 $2^{\mathrm{fl} / \mathrm{f}} / \mathrm{DMSO}$ & $22.9 \pm 0.694^{B}$ & $4.74 \pm 0.279^{B}$ & $0.0716 \pm 0.005^{B}$ & $0.198 \pm 0.016^{8}$ \\
\hline Prx1-Cre Notch2 $2^{\mathrm{f} / f 1} / 3 \mathrm{PO}$ & $12.5 \pm 3.055^{c}$ & $3.31 \pm 0.312^{\mathrm{c}}$ & $0.0629 \pm 0.005^{c}$ & $0.303 \pm 0.034$ \\
\hline
\end{tabular}

Values represent the mean \pm SD. Tb.N, number of trabeculae; Tb.Th, trabecular thickness; Tb.Sp, trabecular spacing. $n \geq 6$ for each group. ${ }^{\mathrm{A}, \mathrm{B}, \mathrm{C} P}<0.05$, for statistically significant effects for ${ }^{\mathrm{A}} 3 \mathrm{PO},{ }^{\mathrm{B}} \mathrm{Cre}$, and ${ }^{\mathrm{C}} 3 \mathrm{PO}-\mathrm{Cre}$ interaction, as determined by 2-way ANOVA.

lated in the bones of PN2 mice, consistent with reduced Notch signaling (31) (Supplemental Figure 1). Western blotting revealed a notable increase in Pfkfb3, Pkm1, Pkm2, and Ldha in the bone protein extracts of PN2 versus their N2 littermates (Figure 2A). To confirm that Notch2 was a major receptor in suppressing glycolysis, we evaluated the effect of Jagged1 on glycolysis in BMSCs from

whereas Ldha helps to sustain glycolysis by regenerating $\mathrm{NAD}^{+}$, while converting pyruvate to lactate. Infection of the BMSCs with Ad-Cre notably reduced Pfkfb3 and Pfkfb4 after 24 or 48 hours and also decreased Ldha levels after 48 hours (Figure 1E). Thus, Notch2 activation concurrently suppresses glycolysis and osteoblast differentiation in bone marrow mesenchymal progenitors.

To corroborate the metabolic regulation by Notch signaling, we activated the pathway in WT BMSCs with recombinant Jagged1 immobilized on the culture dish. After 48 hours of culture, we found that Jagged1 suppressed glucose consumption and lactate secretion when compared with the control Fc (Figure 1F). Biochemically, Jagged1 reduced the abundance of Pfkfb3, Pfkfb4, and Ldha after 24 or 48 hours of culture (Figure 1G). In addition, after 48 hours, Jagged1 suppressed the pyruvate kinases Pkm1 and $\mathrm{Pkm} 2$ that control the last step of glycolysis, without affecting the hexokinase Hk2 (Figure 1G). Thus, as with Notch2 overexpression, Notch activation by Jagged1 suppresses glycolysis in bone marrow mesenchymal progenitors.

Suppression of glycolysis abolishes the excessive bone formation caused by Notch 2 deletion. We next tested the contribution of glycolysis to the effect of Notch signaling on bone formation. We have previously demonstrated that deletion of Notch2 with Prx1-Cre enhances bone formation in the long bones (31). Here, we recreated Prx1-Cre Notch2 ${ }^{\mathrm{f} / \mathrm{l}}$ (PN2) mutant mice by breeding Prx1-Cre Notch $2^{\mathrm{f} / /+}$ males with Notch $2^{\mathrm{f} / \mathrm{l}}$ females. Reverse transcription quantitative PCR (RT-qPCR) analyses with total RNA extracted from cortical bone confirmed that Notch2 mRNA expression was reduced by $50 \%$ in the PN2 mice compared with expression in the Notch $2^{\mathrm{A} / \mathrm{fl}}(\mathrm{N} 2)$ mice (Supplemental Figure 1; supplemental material available online with this article; https:// doi.org/10.1172/JCI96221DS1). On the other hand, Nfatc1, a known target of the Hey transcriptional repressors, was upregu-
$\mathrm{N} 2$ versus those from PN2 mice. While the N2 BMSCs showed an obvious decrease in glucose consumption and lactate secretion in response to Jagged1, the PN2 BMSCs showed no such response (Figure 2B). Thus, Notch2 is a major receptor for mediating the suppression of both bone formation and glycolysis in bone cells.

We next assessed whether increased glycolysis contributes to the excessive bone formation caused by Notch 2 deletion. The small molecule 3PO is a cell-permeable inhibitor of Pfkfb3, which normally produces 2,6-bisphosphofructose, a potent activator of glycolysis (32). We found that treatment of mice with 3PO for 1 month did not significantly change their body weights or cause any obvious distress to the mice (Figure 2C). Treatment with 3PO also did not impair glucose homeostasis, as indicated by the normal fasting blood glucose levels in the mice (Figure 2D). To monitor the potential effect of Notch 2 deletion or $3 \mathrm{PO}$ on glucose metabolism in bone, we performed glucose uptake assays in vivo by injecting ${ }^{14} \mathrm{C}$-labeled 2-deoxyglucose (2-DG). The results showed a clear increase in 2-DG uptake in the bones of PN2 mice compared with uptake in $\mathrm{N} 2$ mice under the control conditions (DMSO injection). Moreover, the month-long 3PO treatment significantly reduced 2-DG uptake in the bones of both PN2 and N2 mice (Figure 2E). Importantly, 3PO significantly reduced the cancellous bone mass in both $\mathrm{N} 2$ and PN2 mice (Figure 2F). As a result, 3PO completely abolished the high-bone-mass phenotype in PN2 mice and rendered the cancellous bone mass indistinguishable between the 2 genotypes (Figure $2 \mathrm{G}$ and Table 1). $3 \mathrm{PO}$ also reduced cortical bone volume without affecting tissue volume in both $\mathrm{N} 2$ and PN2 mice (Table 2). Dynamic histomorphometry revealed that $3 \mathrm{PO}$ reduced both the mineral apposite rate (MAR) and the mineralizing surface over bone surface (MS/BS) percentage, regardless of genotype, thereby completely eliminating the significant increase in bone formation rate over bone surface (BFR/BS) caused by Notch2 deletion

\section{Table 2. $\mu \mathrm{CT}$ analyses of cortical bone in proximal tibiae}

\begin{tabular}{|c|c|c|c|c|c|}
\hline Genotype & BV/TV (\%) & $\operatorname{Tt} . A r\left(m^{2}\right)$ & Ct.Ar $\left(\mathrm{mm}^{2}\right)$ & Ct.Ar/Tt.Ar (\%) & Cortical ThA (mm) \\
\hline Notch2 $2^{f / / f 1} / D M S O$ & $62.6 \pm 2.2$ & $0.94 \pm 0.09$ & $0.59 \pm 0.04$ & $62.6 \pm 2.2$ & $0.20 \pm 0.007$ \\
\hline Notch $2^{\text {fl/fit/3PO }}$ & $58.6 \pm 2.6^{A}$ & $0.76 \pm 0.10$ & $0.45 \pm 0.07^{A}$ & $58.6 \pm 2.6^{A}$ & $0.17 \pm 0.018^{A}$ \\
\hline Prx1-Cre Notch2 2//fi/DMSO & $64.8 \pm 2.1$ & $0.90 \pm 0.07$ & $0.58 \pm 0.04$ & $64.8 \pm 2.2$ & $0.21 \pm 0.010$ \\
\hline
\end{tabular}

Values represent the mean \pm SD. Tt.Ar, total area; Ct.Ar, cortical area; Cortical Th, cortical thickness. $n \geq 6$ for each group. ${ }^{A, B} P<0.05$, for statistically significant effects for ${ }^{\mathrm{A}} 3 \mathrm{PO}$ and ${ }^{\mathrm{B}} 3 \mathrm{PO}$-Cre interaction, as determined by 2 -way ANOVA. 
A

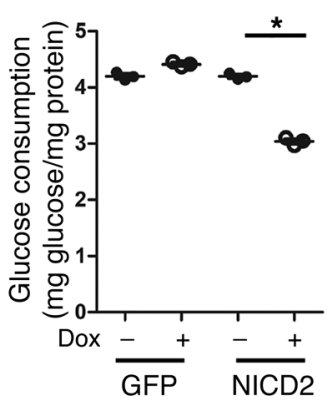

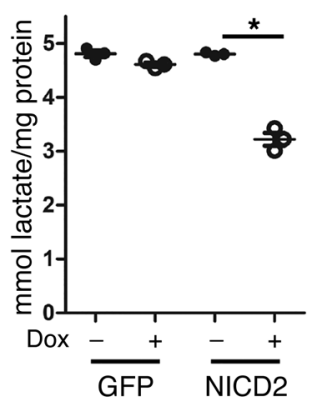
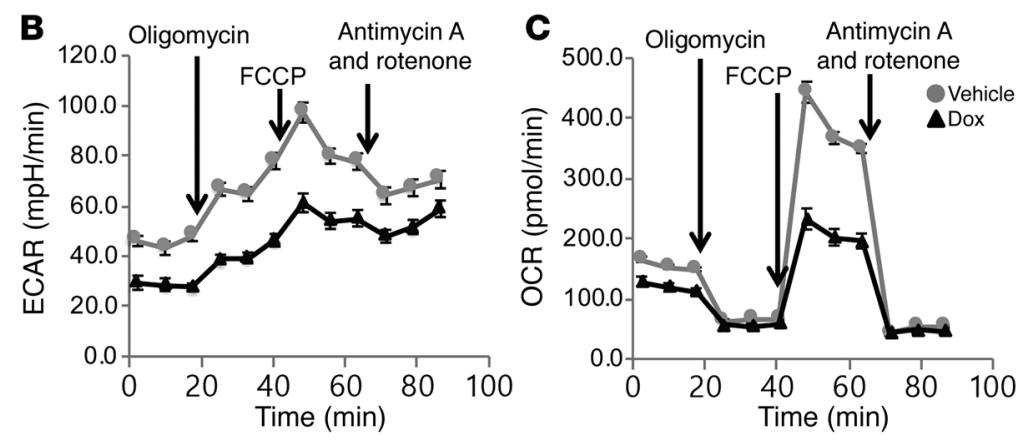
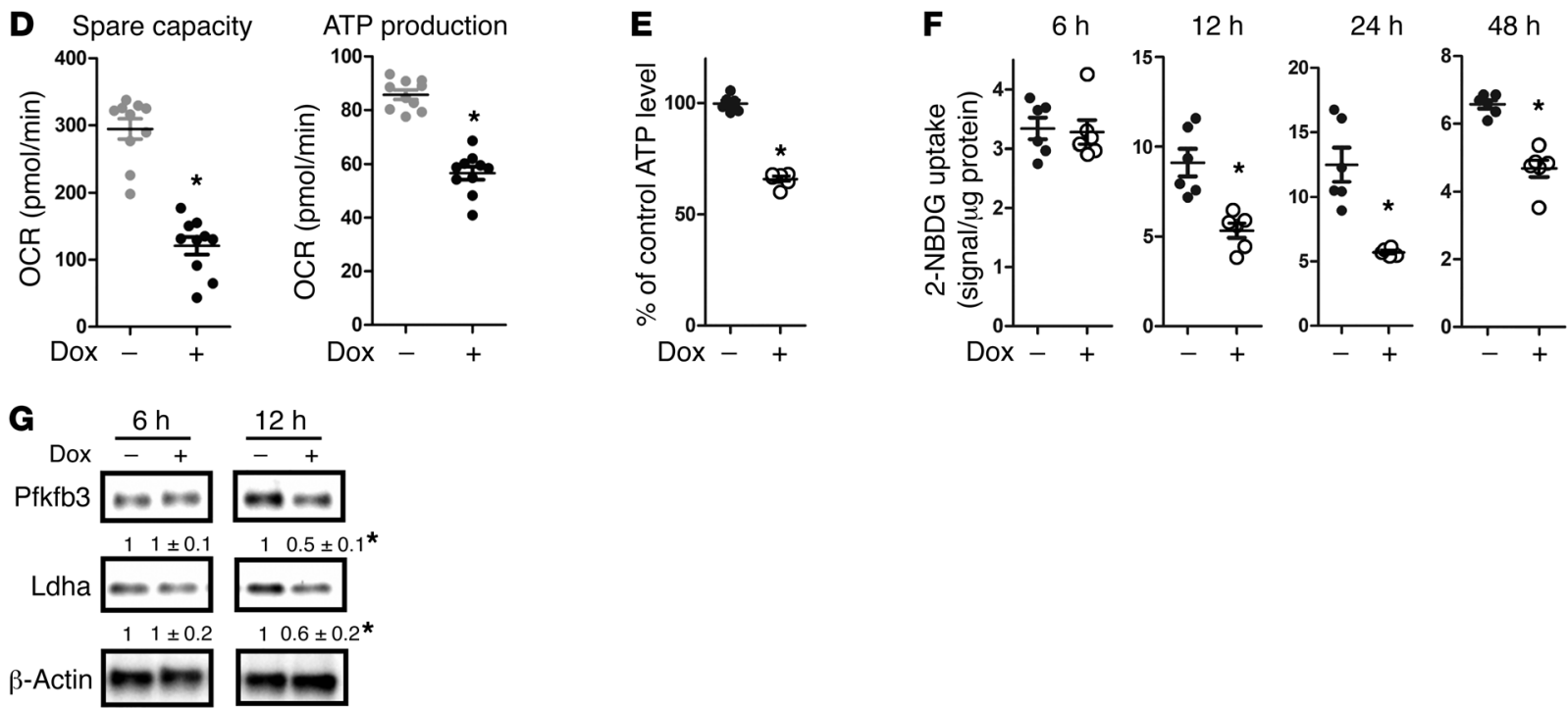

Figure 3. Notch2 signaling suppresses glycolysis in ST2 cells. (A) Glucose consumption and lactate production by GFP-ST2 or NICD2-ST2 cells treated with vehicle or Dox for 48 hours. $n=3$. (B-D) Seahorse analyses of NICD2-ST2 cells treated with vehicle or Dox for 48 hours. $n=10$. (E) Relative ATP levels in NICD2-ST2 cells treated with vehicle or Dox for 48 hours. $n=6$. (F) Glucose uptake assays in NICD2-ST2 cells treated with vehicle or Dox for the indicated durations. $n=6$. (G) Western blots of NICD2-ST2 cells treated with vehicle or Dox for the indicated durations. $\beta$-Actin was used for normalization and quantification (mean \pm SD) was determined from 3 independent samples. After normalization to $\beta$-Actin, the levels in "-Dox" lanes were designated 1 and those in the "+Dox" lanes were further normalized. ${ }^{*} P<0.05$, by 2 -tailed Student's $t$ test (A and $\mathbf{D}-\mathbf{G}$ ).

(Figure 2H). On the other hand, 3PO did not significantly increase the levels of the C-terminal telopeptide of type I collagen (CTX-I) in the serum of either $\mathrm{N} 2$ or $\mathrm{PN} 2$ mice, indicating a relatively small effect on bone resorption (Figure 2I). To confirm the direct effect of $3 \mathrm{PO}$ on osteoblast differentiation, we cultured primary BMSCs from PN2 and N2 mice on Jagged1-treated plates in osteogenic media with or without 3PO. Jagged1 suppressed the expression of the osteoblast markers Alpl, Ibsp, Runx2, and Bglap in N2 BMSCs but did not have a similar effect on PN2 BMSCs (Supplemental Figure 2). In fact, Jagged1 resulted in a slight increase in Alpl and Ibsp mRNA expression in the PN2 BMSCs for reasons unknown at present. Importantly, 3PO suppressed the expression of all markers in both N2 and PN2 BMSCs cultured with Jagged1, indicating that Pfkfb3 functionally interacts with Notch in regulating osteoblast differentiation in vitro (Supplemental Figure 2). Thus, both in vivo and in vitro evidence identifies Pfkfb3 as a key mediator for the suppression of osteoblast differentiation by Notch.

Canonical Notch signaling suppresses glycolysis. To gain further insights into the regulation of glucose metabolism, we chose to activate Notch signaling in ST2 cells, a bone marrow mesenchy- mal progenitor cell line derived from adult mice. The choice of the cell line allowed us to establish stable clones expressing either NICD2 or GFP (termed NICD2-ST2 or GFP-ST2 cells, respectively) in response to doxycycline (Dox). Expression of NICD2, but not GFP, reduced both glucose consumption and lactate secretion by ST 2 cells after 48 hours of Dox induction (Figure 3A). Seahorse analyses confirmed that induction of NICD2 for 48 hours suppressed the extracellular acidification rate (ECAR), with or without the mitochondria stressors (Figure 3B). NICD2 also reduced the oxygen consumption rate (OCR) in both basal conditions and in response to the uncoupling reagent FCCP (Figure 3C). Quantification of the OCR data revealed a decrease in both ATP production from mitochondrial respiration and the spare respiratory capacity upon NICD2 expression (Figure 3D). Biochemical measurements revealed a notable reduction in steady-state intracellular ATP levels in response to 48 hours of Dox treatment (Figure $3 \mathrm{E})$. In keeping with reduced glucose consumption, incubation of the cells with the fluorescent glucose analog 2-NBDG revealed that NICD2 began to suppress glucose uptake after 12 hours of Dox treatment (Figure 3F). Western blot analyses showed that 

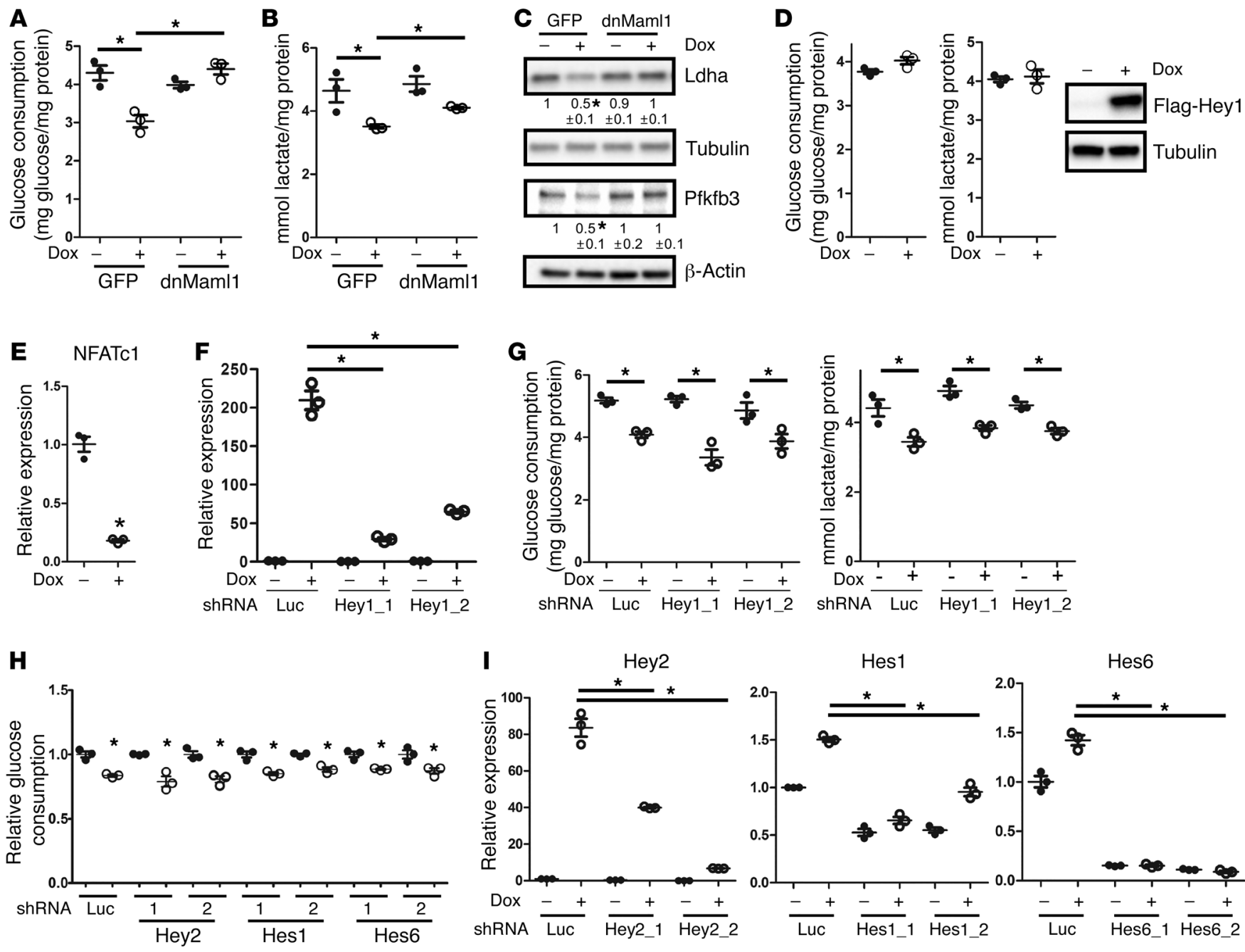

Figure 4. Canonical Notch signaling suppresses glycolysis. (A-C) Effects of retroviral expression of GFP or dnMaml1 on glucose consumption (A), lactate production (B) or metabolic enzymes (C) in NICD2-ST2 cells treated with vehicle or Dox for 48 hours. $\beta$-Actin was used for normalization, and quantification (mean \pm SD) was determined from 3 independent samples. After normalization to $\beta$-Actin, the level in the left-most lane is designated 1 , and those of the other lanes are further normalized to the left-most lane. (D) Glucose consumption and lactate production in Hey1-ST2 cells treated with vehicle or Dox for 48 hours. Western blot shows the induction of Flag-Hey1 by Dox. $n=3$. (E) Relative mRNA levels of NFATc1 by RT-qPCR in Hey1-ST2 cells treated with vehicle or Dox for 48 hours. $n=3$. (F) RT-qPCR analyses of Hey1 in NICD2-ST2 cells infected with lentivirus expressing 2 different Hey1 shRNAs or the control luciferase shRNA (shLuc). (G) Glucose consumption and lactate production in NICD2-ST2 cells infected with lentivirus expressing different shRNAs, with or without Dox for 48 hours. (H) Relative glucose consumption in NICD2-ST2 cells infected with lentivirus expressing different shRNAs, with or without Dox for 48 hours. Glucose consumption was normalized to the protein amount and then to the vehicle-treated group designated as 1 . (I) RT-qPCR analyses of mRNA levels showing shRNA knockdown efficiency. $n=3$. ${ }^{*} P=0.05$, by 2-way ANOVA followed by Bonferroni's post hoc test (A, B, F, and I) or 2-tailed Student's $t$ test (C, E, G, and $\mathbf{H})$.

NICD2 reduced both Pfkfb3 and Ldha after 12 hours of induction (Figure 3G). Thus, as with Notch activation in the primary bone marrow mesenchymal cells, sustained Notch2 signaling in ST2 cells suppressed glycolysis.

We next assessed the potential role of canonical Notch signaling in the regulation of metabolism. Specifically, we tested the effect of dominant-negative Maml1 (dnMaml1), which has been well established as an inhibitor of the canonical Notch signaling pathway (33). Retroviral expression of dnMaml1, but not GFP, completely eliminated the suppression of glucose consumption by NICD2 and partially restored the production of lactate (Figure 4, A and B). Consistent with metabolic rescue, dnMaml1 restored Pfkfb3 and Ldha expression to normal levels in response to NICD2 (Figure 4C). It is worth noting that dnMaml1 alone did not affect glucose consumption, lactate production, or the metabolic enzymes, confirming the specific antagonism of dnMaml1 on Notch2 signaling. Thus, Notch2 signaling suppresses glucose metabolism through a Maml1-dependent mechanism.

Given that the Hes/Hey transcriptional repressors are the prototypical mediators of canonical Notch signaling, we examined whether they were responsible for metabolic regulation. We focused on Hey1, as our earlier results showed that it was the most robustly induced by NICD2 among the Hes and Hey genes in BMSCs (Figure 1A). To this end, we generated a stable ST2 cell line expressing Hey1 in response to Dox. However, Hey1 expression did not affect glucose consumption or lactate production, 

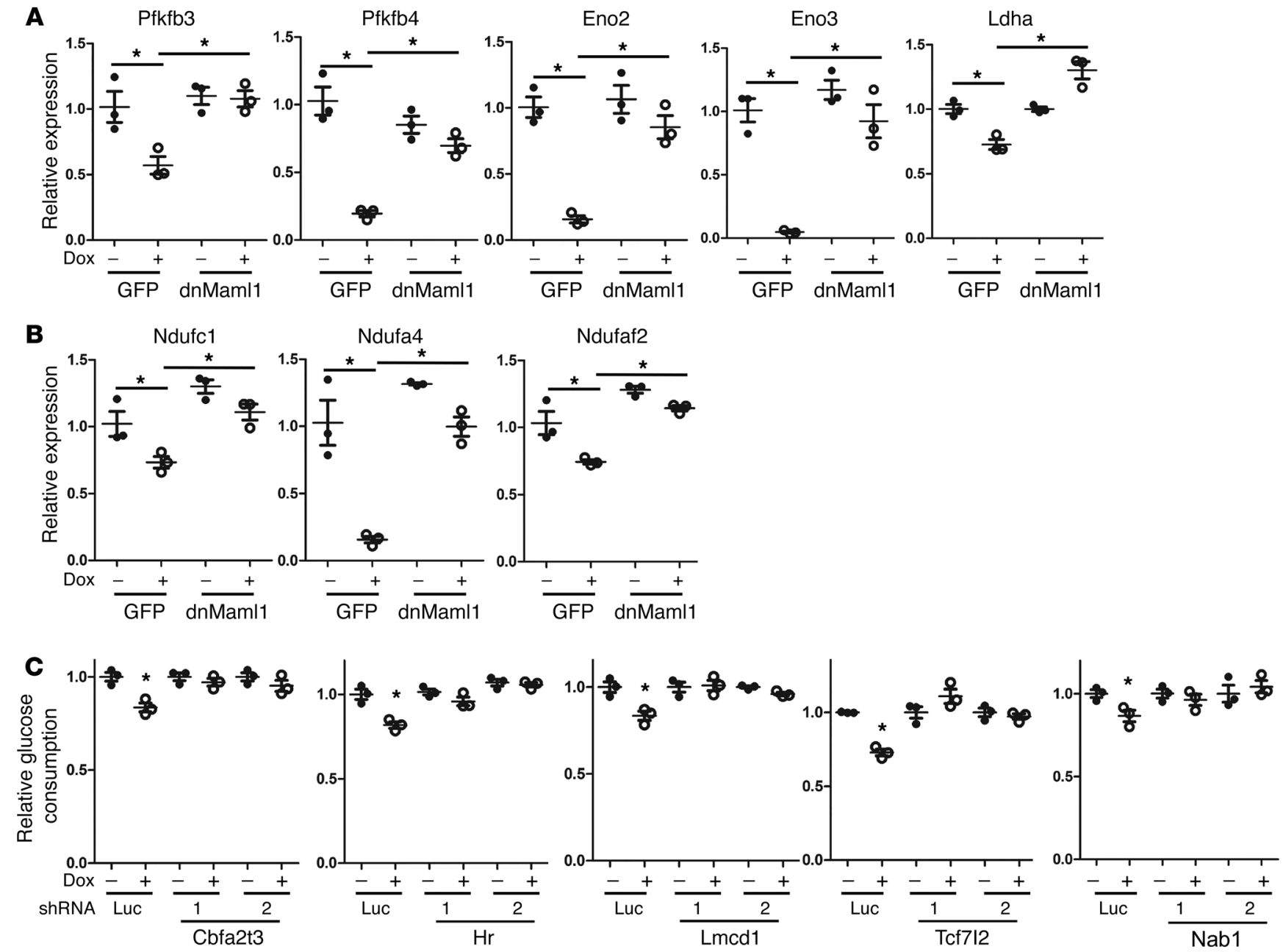

Figure 5. Canonical Notch signaling suppresses gene expression of glycolytic enzymes and components of the mitochondrial ETC. (A and B) Relative mRNA levels of glycolytic enzymes (A) and components of the mitochondrial ETC (B) in NICD2-ST2 cells with retroviral expression of GFP or dnMaml1 and treatment with vehicle or Dox for 24 hours. (C) Relative glucose consumption in NICD2-ST2 cells infected with lentivirus expressing different shRNAs, as indicated, with or without Dox for 48 hours. Glucose consumption was normalized to the protein amount and then to the no-Dox control designated as 1 . $n=3$. ${ }^{*} P<0.05$, by 2-way ANOVA followed by Bonferroni's post hoc test (A and B) or 2-tailed Student's $t$ test (C).

even though it suppressed the mRNA levels of NFATc1, which was previously identified as a Hey1 target gene (Figure 4, D and E) (31). To further test the potential relevance of Hey1, we knocked down its expression in NICD2-ST2 cells with shRNA expressed from a lentiviral vector. Whereas Dox markedly induced Hey1 in cells expressing the control shRNA targeting the luciferase gene (shLuc), 2 independent Hey1 shRNA constructs (Hey1_1 and Hey1_2) diminished the induction of Hey1 by more than $80 \%$ (Figure 4F). However, neither construct showed any effect on the suppression of glucose consumption or lactate secretion by Dox in NICD2-ST2 cells (Figure 4G). Therefore, induction of the Hes/ Hey proteins does not appear to be the main mechanism mediating metabolic regulation by Notch.

To gain further insights into the mechanism underlying glycolytic suppression by Notch, we performed RNA-Seq experiments using NICD2-ST2 cells with or without Dox treatment for 24 hours. Among the genes with mRNA levels changed by a minimum of $20 \%$, the prototypical Notch target genes, including Hes1, Hes6, Hey1 and Hey2, were induced by NICD2. However, like that of
Hey1, effective knockdown of Hey2, Hes1, or Hes6 did not alleviate the suppression of glucose consumption caused by NICD2 overexpression (Figure 4, H and I). Importantly, several suppressed genes encode glycolytic enzymes including Pfkfb2, Pfkfb3, Pfkfb4, Eno2, Eno3, and Ldha, while several others, such as Ndufaf2, Ndufc1, Ndufs5, and Ndufaf4 of complex I, and Ndufa4 of complex IV, code for components of the mitochondrial electron transport chain (ETC). RT-qPCR experiments confirmed the suppression of those genes by NICD2 (Supplemental Figure 3). Furthermore, dnMaml1, which was shown earlier to negate the metabolic effect of Notch, either abolished or alleviated the suppression of glycolytic or ETC genes by NICD2 (Figure 5, A and B). Thus, transcriptional suppression of both glycolytic and mitochondrial ETC genes may contribute to the restriction of glycolysis by Notch.

To explore the mechanism whereby canonical Notch signaling suppresses metabolic gene expression, we investigated the potential role of transcriptional repressors beyond the Hes/Hey family. RNA-Seq experiments identified 14 additional known repressors that were induced by at least 2-fold and that had an 
Table 3. Role of transcriptional suppressors in glycolytic suppression by Notch

\begin{tabular}{|c|c|}
\hline Gene name & Role \\
\hline Cbfa2t3 & Yes \\
\hline Hr & Yes \\
\hline Lmcd1 & Yes \\
\hline Tcf7l2 & Yes \\
\hline Nab1 & Yes \\
\hline Hey1 & No \\
\hline Hey2 & No \\
\hline Hes1 & No \\
\hline Hes6 & No \\
\hline Msx1 & No \\
\hline Zfp703 & No \\
\hline Foxs1 & No \\
\hline Nfatc4 & No \\
\hline Btg2 & No \\
\hline Cobra1 & No \\
\hline Brms1 & No \\
\hline Tle1 & No \\
\hline Bach1 & No \\
\hline
\end{tabular}

induced level of more than 10 reads per kb per million mapped reads (RPKM) upon NICD2 expression over a 24-hour period in ST2 cells. RT-qPCR experiments confirmed the induction of the repressors by NICD2 after 24 hours and, further, that their induction was abolished by the viral expression of dnMaml1. We then systematically knocked down each repressor with 2 independent shRNAs in NICD2-ST2 cells and measured glucose consumption with or without Dox induction over a 48-hour period. The results showed that knockdown of Cbfa2t3, Hr, Lmcd1, Tcf7l2, or Nab1 essentially eliminated glycolytic suppression by NICD2, whereas the other repressors did not have the same effect (Figure 5C and Supplemental Figure 4) (Table 3). Thus, it is likely that canonical Notch signaling induces the expression of multiple transcriptional repressors to suppress glycolysis.

Notch suppresses glycolysis through downregulation of AMPK activity. The suppression of complex I genes by Notch2 prompted us to examine the potential role of complex I in metabolic regulation. NICD2 expression in response to Dox for 24 hours reduced the activity of complex I in NICD2-ST2 cells (Figure 6A). Consistent with complex I being a major producer of mitochondrial superoxide, we found that NICD2 decreased the level of mitochondrial superoxide after 12 or 24 hours of Dox induction (Figure $6 \mathrm{~B})$. These results are consistent with our earlier observation that NICD2 expression reduced overall oxygen consumption (Figure 3C). Overall, Notch2 signaling suppresses mitochondrial respiration and the production of ROS.

Recent studies have demonstrated that mitochondrial superoxide activates adenosine 5 -monophosphate-activated protein kinase (AMPK) through $\mathrm{H}_{2} \mathrm{O}_{2}$ (34). Because of the essential role of AMPK in cellular metabolism, we examined its potential relevance to glycolytic suppression by Notch. As a heterotrimeric kinase composed of $\alpha, \beta$, and $\gamma$ subunits, AMPK activation correlates with phosphorylation at Thr172 on the $\alpha$ subunit (35). We found that phosphorylation at Thr172 was reduced in ST2 cells upon NICD2 expression for 12 or 48 hours and observed the same kinetics as that for the suppression of glucose uptake (Figure 6C, compare with Figure 3F). Importantly, dnMaml1, shown above to abolish the metabolic effect of NICD2, also restored the phosphorylation of AMPK and acetyl-coA carboxylase (ACC), a prototypical substrate of AMPK (Figure 6, D and E). The suppression of AMPK by Notch was confirmed in WT BMSCs, as Jagged1 reduced the levels of phosphorylated AMPK $\alpha$ (p-AMPK $\alpha$ ) and p-ACC (Figure 6F). To test the functional relevance of AMPK suppression to Notch function, we force-activated the enzyme with the AMP analog AICAR. At either 50 or $100 \mu \mathrm{M}$, AICAR did not affect basal glucose consumption or lactate secretion by BMSCs but abolished the suppression by Jagged1 (Figure 6G). Likewise, $100 \mu \mathrm{M}$ AICAR overcame the suppression of glucose consumption and lactate secretion by Dox-induced NICD2 expression in the NICD2-ST2 cells (Figure 7A). However, AICAR had little to no effect on mRNA levels of the glycolytic genes that were suppressed by NICD2 (Figure 7B). Thus, we found that force-activation of AMPK was sufficient to counteract the glycolytic suppression by Notch, without affecting glycolytic gene expression. This observation is consistent with previous reports that AMPK phosphorylates Pfkfb3 to increase its enzymatic activity and hence glycolysis. Finally, to test whether the suppression of complex I genes was sufficient to reduce AMPK activity, we knocked down Ndufaf2, which was suppressed by Notch, and assayed for AMPK phosphorylation. We found that 2 independent shRNAs effectively reduced Ndufaf 2 mRNA expression and decreased p-AMPK $\alpha$ levels relative to total AMPK levels (Figure 7C). Taken together, the results support a model in which canonical Notch signaling induces multiple transcriptional repressors to suppress both glycolytic and mitochondrial ETC genes. Whereas the former reduces the abundance of glycolytic enzymes, the latter results in decreased AMPK activity that probably restricts the activity of the enzymes (Figure 8).

\section{Discussion}

We have provided evidence that Notch signaling suppresses glucose metabolism in mesenchymal progenitors to inhibit osteoblast differentiation. The mechanism of the metabolic regulation by Notch involves the transcriptional downregulation of multiple metabolic genes integral to glycolysis or the mitochondrial ETC. The decrease in mitochondrial respiration in turn reduces ROS production and AMPK activity, resulting in further suppression of glycolysis. We believe that these results shed new light on the mechanism through which Notch regulates bone physiology.

Exactly how Notch signaling suppresses metabolic genes is not clear at present. The data clearly indicate the importance of the Maml proteins, thus implicating canonical Notch signaling in metabolic regulation. However, since the NICD-Maml-Rbpjk complex is known to activate gene expression, the gene suppression we observed must be indirect. Although Hes/Hey transcription suppressors are prototypical mediators for Notch, they are unlikely to be the main driver for metabolic suppression, as overexpression of Hey1 did not impair glycolysis, and knockdown of the relevant Hes/Hey genes did not blunt the Notch effect. On the other hand, 5 transcriptional repressors induced by NICD2 contributed to the glycolytic suppression, but future experiments are 

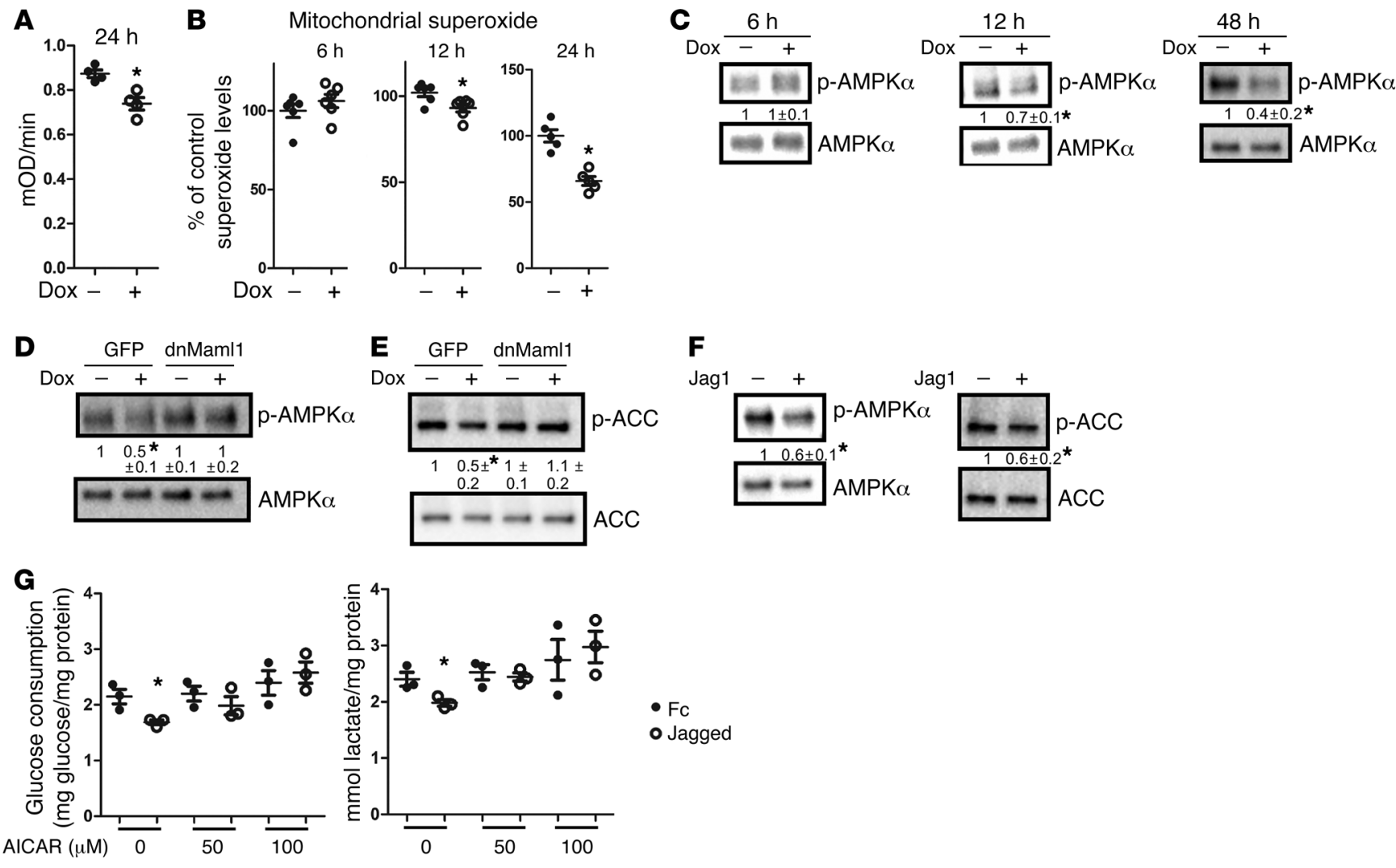

Figure 6. Notch suppresses glycolysis through downregulation of AMPK activity. (A-C) Measurements of mitochondrial complex I activity $(n=4)(\mathbf{A})$, mitochondrial superoxide levels $(n=6)(B)$, and p-AMPK $\alpha$ levels $(n=3)(C)$ in NICD2-ST2 cells treated with vehicle or Dox for the indicated durations. Total AMPK $\alpha$ was used for normalization, and quantification (mean \pm SD) was determined from 3 independent samples. After normalization to total AMPK $\alpha$, the level in the left lane was designated 1 and that of the right lane was further normalized to the left lane. mOD, mitochondrial OD. (D and E) Western blot analyses of NICD2-ST2 cells with retroviral expression of GFP or dnMaml1 and treatment with vehicle or Dox for 24 hours. Phosphorylation levels were normalized to total AMPK $\alpha$ or ACC, and quantification (mean \pm SD) was determined from 3 independent samples. After normalization to total protein, the level in the left-most lane was designated 1 and the other lanes were further normalized to that lane. (F) Western blots of BMSCs with or without Jagged1 stimulation for 24 hours. Normalization and quantification were determined as in $\mathbf{D}$ and $\mathbf{E}$. (C) Effects of AICAR at the indicated concentration for 48 hours on glucose consumption and lactate production in BMSCs with or without Jagged1 stimulation. $n=3$. ${ }^{*} P<0.05$, by 2 -tailed Student's $t$ test (A-C).

necessary to establish the potential direct suppression of glycolytic or ETC genes by any of the repressors implicated here.

The current study highlights the fact that metabolic regulation by Notch is highly dependent on cellular context. A previous study has shown that Notch induces the transcription of Glut1, hexokinase A (Hex-A), and Impl3 (Ldha homolog) in Drosophila cells, resulting in a Warburg-like effect (27). However, RNA-Seq detected no change in Glut1, Hk1, or Hk2 upon expression of NICD2 in ST2 cells. On the contrary, we observed a downregulation of Ldha and other key glycolytic enzymes and, consequently, a suppression of glycolysis by Notch. Another study using mouse macrophages indicated that Notch stimulates mitochondrial oxidative phosphorylation in part through the transcriptional induction of pyruvate dehydrogenase phosphatase catalytic subunit 1 (Ppd1) to promote the M1 phenotype (29). However, we observed a notable decrease in both Pdp1 (-1.7-fold) and Pdp2 (-2.6-fold) in ST2 cells after 24 hours of NICD2 expression. On the other hand, our finding about the suppressive role of Notch in glucose metabolism is reminiscent of that previously reported in endothelial cells, in which Notch reduced Pfkfb3 and glycolytic flux (28). Overall, although the exact reason for the different effects of Notch on glu- cose metabolism is not known, they likely reflect the specific epigenetic landscape and signaling network in each cell type.

The current study further strengthens the link between bone formation and cellular metabolism. Previous studies have demonstrated that Wnt, PTH, and Igf signaling stimulates glycolysis and glutamine catabolism while increasing bone accrual (36). Here, Notch suppressed osteoblast differentiation, at least in part through the restriction of glucose metabolism. Together, these findings indicate that metabolic rewiring plays an integral role in the regulation of bone formation and, further, that targeting metabolic pathways may be a promising avenue for the development of novel bone therapies.

\section{Methods}

Mouse strains. Notch2 ${ }^{\mathrm{f} / \mathrm{l}}, \mathrm{R} 26-\mathrm{NICD} 2$, and Prx1-Cre mouse strains were generated as previously described $(30,37,38)$. We thank Ryuichi Nishinakamura (Kumamoto University, Japan) for providing the R26NICD2 mouse strain. All mice were housed in a specific pathogen-free (SPF) barrier facility managed by the Department of Comparative Medicine of Washington University. The animals were group housed under a 12-hour light cycle (6 am-6 pm) and fed standard chow (PicoLab Mouse Diet 20, product 5058). 

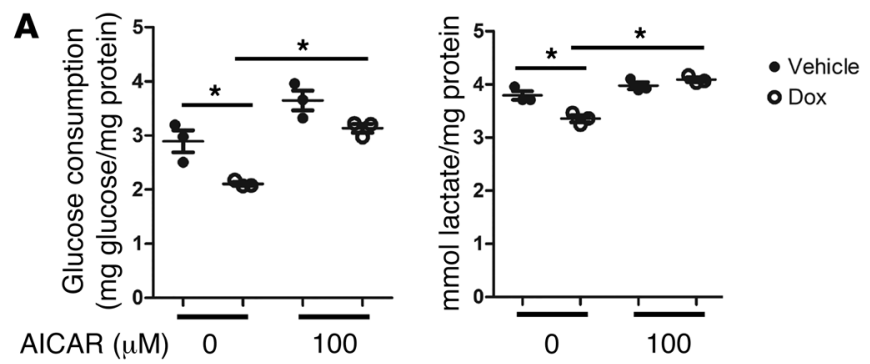

B
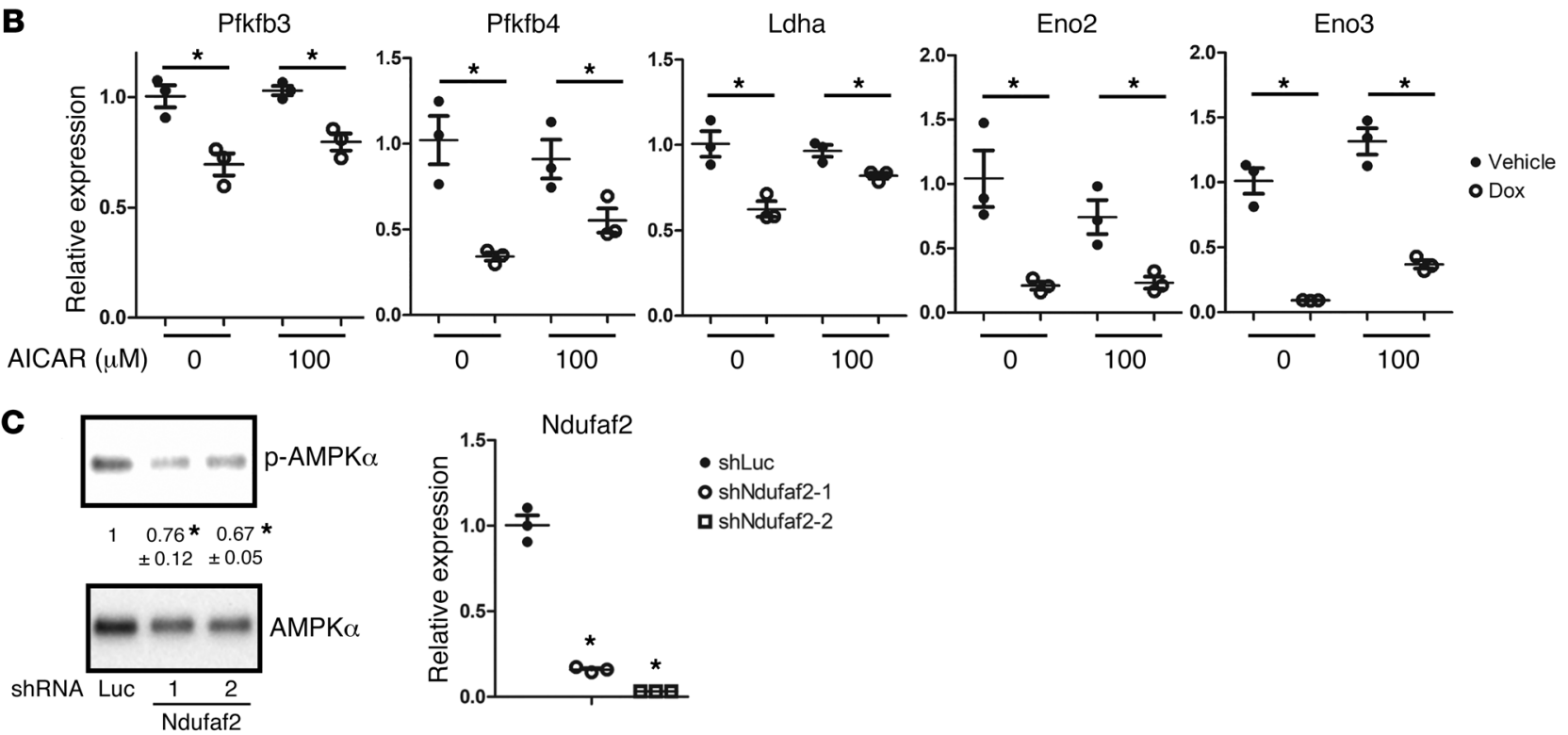

Figure 7. AMPK activation rescues metabolic inhibition by Notch2 without restoring glycolytic gene expression. (A) Effects of AICAR for 48 hours on glucose consumption and lactate production in NICD2-ST2 cells. (B) RT-qPCR analyses of relative mRNA levels of glycolytic genes. (C) Western blot and RTqPCR analyses of ST2 cells infected with lentivirus expressing shRNAs targeting luciferase (Luc) or Ndufaf2. pAMPK was first normalized to total AMPK in each lane. The normalized level in the left -most lane was designated 1 and those of the other lanes were further normalized to that lane. All RT-qPCR data were normalized to 185 rRNA. $n=3 .{ }^{*} P<0.05$, by 2-way ANOVA followed by Bonferroni's post hoc test (A) or 2-tailed Student's $t$ test (B and $\mathbf{C}$ ).

Analyses of bone. For 3PO treatment, mice were injected i.p. with the chemical (MilliporeSigma, 525330) dissolved in DMSO at $50 \mathrm{mg} /$ $\mathrm{kg}$ body weight or an equal volume of DMSO as a control daily beginning on P24 for 4 weeks. Micro-CT ( $\mu$ CT 40, Scanco Medical AG) was performed on tibiae. Quantification of the cancellous bone was assessed by measuring $100 \mu \mathrm{CT}$ slices $(1.6 \mathrm{~mm})$ immediately below the growth plate, with a threshold of 200. For cortical bone parameters, $50 \mu \mathrm{CT}$ slices $(0.8 \mathrm{~mm})$ from the mid-shaft of the tibia were analyzed, with a threshold of 260.

For dynamic histomorphometry, calcein (MilliporeSigma) dissolved in water ( $\mathrm{pH}$ 7.2-7.4, adjusted with $\mathrm{NaOH}$ ) was injected i.p. at $7.5 \mathrm{mg} / \mathrm{kg}$ body weight on days 7 and 2, respectively, prior to sacrifice. Bones were fixed in $70 \%$ ethanol, embedded in methyl-methacrylate, and sectioned at $10-\mu \mathrm{m}$ thickness. Histomorphometric parameters were acquired with a BIOQUANT Osteo II from 3 sections per mouse and 3 mice for each genotype.

In vivo glucose uptake assay. Mice injected with $3 \mathrm{PO}$ or DMSO vehicle for 4 weeks were first examined for serum glucose levels. For this, blood samples were collected through the tail vein after the mice were starved for 3 hours, and blood glucose levels were measured with a glucose meter (Ascensia Contour; Bayer). The mice were rested for 30 minutes before being injected i.p. with ${ }^{14} \mathrm{C}-2 \mathrm{DG}$ (PerkinElmer,
NEC495A001MC) at $10 \mu \mathrm{Ci}$ per $20 \mathrm{~g}$ body weight and then sacrificed after 2 hours for collection of tibiae and femurs. The growth plates were surgically removed with a scalpel under the microscope before the bone marrow was cleared through centrifugation. The remaining trabecular and cortical bone was weighed, chopped with surgical scissors, and then lysed in RIPA buffer for 30 minutes on ice. After centrifugation, the supernatant was collected for scintillation counting.

Serum biochemical assays. For serum CTX-I or P1NP assays, serum was collected through retro-orbital bleeding from mice starved for 6 hours, and the serum was analyzed with the RatLaps ELISA Kit (Immunodiagnostic Systems Ltd., AC-06F1) or the Rat/Mouse PINP EIA Kit (Immunodiagnostic Systems Ltd., AC-33F1) according to the manufacturers' instructions.

Western blot analysis. ST2 cells or mouse primary BMSCs were harvested in lysis buffer (50 mM TrisCl, $150 \mathrm{mM} \mathrm{NaCl}, 1 \% \mathrm{NP}-40,5 \mathrm{mM}$ EDTA, 5\% glycerol) containing proteinase inhibitors and phosphatase inhibitors (Thermo Fisher Scientific, catalog 78442). To extract bone proteins, femurs and tibiae were pulverized in liquid nitrogen after surgical removal of the epiphysis and flushing of the marrow. The bone powders were harvested in the lysis buffer and centrifuged to collect the soluble proteins that were later quantified with the Micro BCA Protein Assay Kit (Thermo Fisher Scientific). Total protein $(10 \mu \mathrm{g})$ 


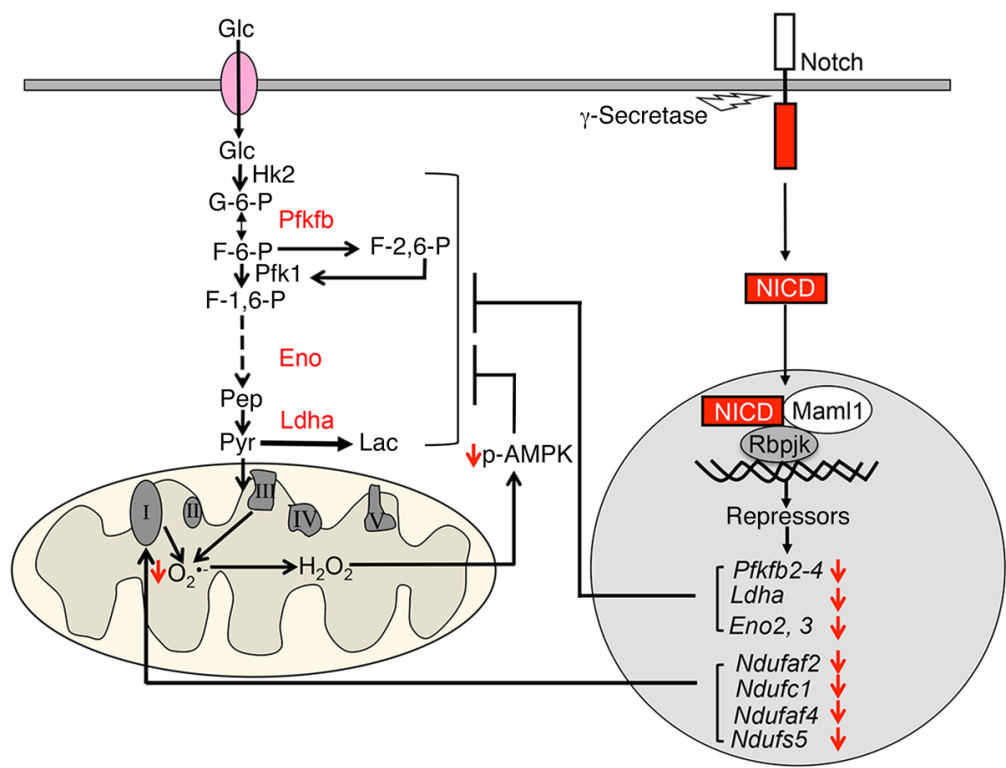

Figure 8. Working model for Notch signaling in the suppression of glucose metabolism in mesenchymal progenitors. Canonical Notch signaling induces multiple transcriptional repressors that suppress the transcription of genes encoding glycolytic enzymes or mitochondrial complex I components. The decrease in mitochondrial respiration lowers AMPK activity and helps to reduce glycolysis. was resolved by electrophoresis on Mini-PROTEAN TGX Precast Gel (Bio-Rad), transferred onto a $0.45-\mu \mathrm{m}$ pore PVDF Immobilon-P membrane (MilliporeSigma), and detected with a specific antibody against Ldha (product no. 2012), AMPK $\alpha$ (product no. 2531), p-AMPKa (product no. 2532), $\beta$-actin (product no. 4970), Pkm2 (product no. 4053), p-ACC (product no. 3661), or ACC (product no. 3676) (all purchased from Cell Signaling Technology); or an antibody against Pfkfb4 (product no. PAB4031) from Novus Biologicals; or Pkm1 (product no. SAB4200094) from MilliporeSigma. Membranes were blocked for 1 hour at room temperature in 5\% BSA (Roche) or 5\% milk (Bio-Rad) in TBS containing 0.1\% Tween-20 (TBST), followed by overnight incubation at $4^{\circ} \mathrm{C}$ with the primary antibody. Then, the membranes were washed with TBST and further incubated at room temperature for 1.5 hours with an HRP-conjugated anti-rabbit (GE Healthcare, product no. NA934V) or anti-mouse (Cell Signaling Technology, product no. 7076S) secondary antibody. All blots were developed using the Clarity Substrate Kit (Bio-Rad). Gel images were captured with ChemiDoc (Bio-Rad). Each experiment was repeated with a minimum of 3 independently prepared protein samples.

Generation of Dox-inducible stable cell lines. To generate the TetOFUW-FlagNICD2-IRES-EGFP lentiviral construct, we first created the vector TetO-FUW-polylinker by replacing cMYC in the TetO-FUWcMYC plasmid (Addgene plasmid 20324) with a polylinker containing EcoRI, AscI, AsisI, AfeI, PacI, SwaI, SbfI, and XbaI. We then released 3xFlag-NICD2 from p3xFlag-CMV-NICD2(Met1669-Ala2470) with EcoRI and SmaI and inserted it into the EcoRI and AfeI sites of TetOFUW-polylinker (39). Subsequently, IRES-EGFP amplified by PCR from pCIG was inserted into the PacI and XbaI sites of the polylinker to produce the final construct TetO-FUW-FlagNICD2-IRES-EGFP (40).

To make the TetO-FUW-FlagHey1-IRES-EGFP plasmid, we first inserted Hey1 cDNA into the XbaI and BamHI sites of p3xFlagCMV-7 (MilliporeSigma) to produce p3xFlag-CMV-Hey1. We then released 3xFlag-Hey1 from p3xFlag-CMV-Hey1 with EcoRI and SmaI and inserted it into the EcoRI and AfeI sites of TetO-FUW-polylinker. Finally, IRES-EGFP was inserted in the same way as described above.

Lentiviruses were produced according to a standard protocol. Briefly, 293T cells were transfected with FUW-M2rtTA (Addgene

plasmid 20342), TetO-FUW-FlagNICD2-IRES-EGFP or TetO-FUWFlagHey1-IRES-EGFP, together with psPAX2 and pMD2.G. After overnight incubation, the cells we placed into fresh media that was then harvested for viruses after 2 days.

To produce the stable cell lines, ST2 cells were coinfected with the FUW-M2rtTA virus, together with either the TetO-FUW-FlagNICD2IRES-EGFP or the TetO-FUW-FlagHey1-IRES-EGFP virus. The cells were then plated at single-cell density in 96-well plates for colony selection. The positive clones were identified on the basis of green fluorescence expression and Western blotting in the presence of Dox.

Cell culture. ST2 cells and BMSCs were grown in aMEM (Gibco, Thermo Fisher Scientific), and 293T cells were grown in DMEM

Table 4. Primers used for RT-qPCR

\begin{tabular}{|c|c|c|}
\hline Cene name & Forward sequence $\left(5^{\prime}-3^{\prime}\right)$ & Reverse sequence $\left(5^{\prime}-3^{\prime}\right)$ \\
\hline Alpl & ACACCAATGTAGCCAAGAATGTCA & GATTCGGGCAGCGGTTACT \\
\hline Bglap & TGCTGGAGTGGTCTCTATCA & АСССТСТTСССАСАСТGT \\
\hline BSP & ССGGCСACGСТACTTTCTT & GGACTGGAAACCGTTTC \\
\hline 185 & CGGCTACCACATCCAAGGAA & GCTGGAATTACCGCGGCT \\
\hline Eno2 & САGСACTTTGTCCGGAACTATC & AGGTCATCGCCCACTATCT \\
\hline Eno3 & GAACTCCGAGATGGAGACAAAG & GGACCTAGAGTCTTGTTGATGTG \\
\hline Hey1 & СACTGCAGGAGGGAAAGGTTAT & ССССАAАСТСССATAGTCCAT \\
\hline Hey2 & САТGTCGССТАТССАСАТСTT & GGCATCCGAAGAGCAGAAT \\
\hline Hes6 & CGGATCAACGAGAGTCTTCAG & СACGGTCAGCTCCACAAC \\
\hline Hes7 & САTCAACCGCAGCCTAGAA & GAACTCCAGTATCTCCGCTTTC \\
\hline Ldha & TGGAAGACAAACTCAAGGGCGACA & TGACCAGCTTGGAGTTCGCAGTTA \\
\hline Ndufa4 & СССАGCTTCATTCCTCTCTTC & GGCTCTGGGTTGTTCTTTCT \\
\hline Ndufaf2 & ACTGGAGAGGGCAGACTATT & GGTGGAGTCTTCCTTGTTCTTC \\
\hline Ndufc1 & САTCTTCATTGTGTGTTTGGATGA & TCAACACGGTCGAAGTTCTATG \\
\hline NFATC1 & GAGACAGACATCGGGAGGAAGA & GTGGGATGTGAACTCGGAAGA \\
\hline Pfkfb2 & GACTGCAACAGCAGCTATAAAC & GCTGGCAAGCCAATCATAAC \\
\hline Pfkfb3 & СТACCTCAACTGGATAGGTGTTC & AGGGCGGAAGAAGTTGTAAG \\
\hline Pfkfb4 & САTCCAGAGTCGCATCGTTTA & стTGAGGTTTAGCTCGCTCTC \\
\hline Sp7 & GGAAAGGAGGCACAAAGAAGC & ССССtTAGGCACTAGGAG \\
\hline
\end{tabular}




\section{Table 5. shRNA targeting sequences}

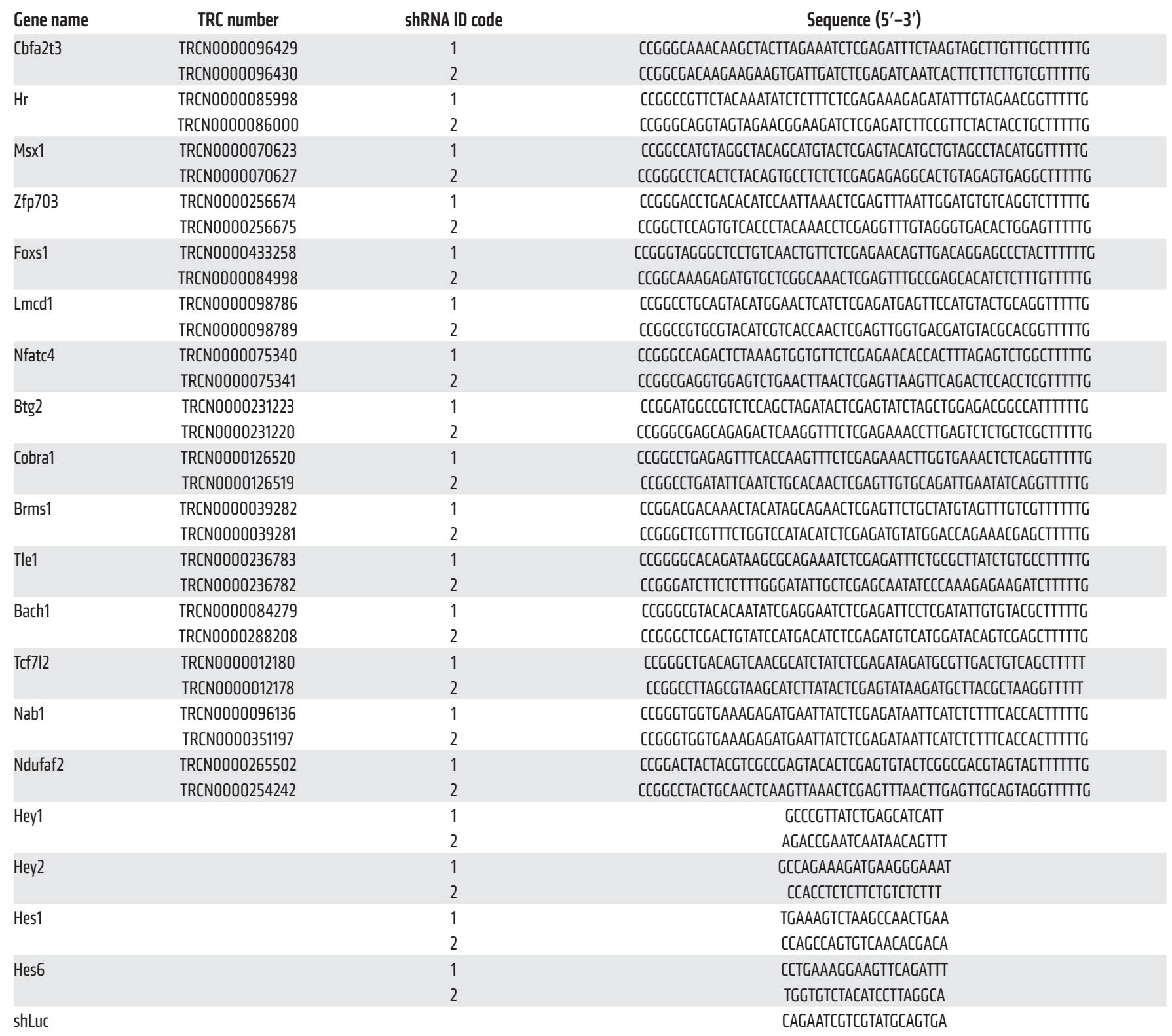

TRC, The RNAi Consortium.

(Gibco, Thermo Fisher Scientific), both supplemented with 10\% heatinactivated FBS and penicillin-streptomycin. BMSCs were isolated from tibiae and femurs of 2-month-old mice. Briefly, the bones were cleanly dissected and the epiphysis cleared off with scissors. Bone marrow cells were collected from the bones by centrifugation and then incubated with RBC lysis buffer (MilliporeSigma, R7757-100ML). The remaining cells were then passed through a $70-\mu \mathrm{m}$ cell strainer before being seeded onto a tissue culture plate in $\alpha$-MEM containing 10\% FBS. After daily changes of the medium for 3 days, the cells were allowed to grow until they reached confluence. The cells were then dissociated and seeded onto 24-well plates for experiments. For the Dox-inducible experiments in ST2 cells, the cells were incubated overnight before treatment with $500 \mathrm{ng} / \mathrm{ml}$ Dox (MilliporeSigma,
D-9891). In some experiments, the cells were infected with dnMaml1or GFP-expressing retroviruses for 8 hours and recovered in regular media overnight before being seeded overnight for Dox treatment. The retroviral plasmids MSCV-DNMAML1-GFP and MSCV-GFP were provided by Jon Aster (Brigham and Women's Hospital, Boston, Massachusetts, USA) (41). To test the effect of Jagged1, the cell culture plates were first coated overnight at room temperature with 50 $\mu \mathrm{g} / \mathrm{ml}$ protein G (Thermo Fisher Scientific, 10-1201) in Dulbecco's phosphate-buffered saline (DPBS) (Life Technologies, Thermo Fisher Scientific). The plates were then washed 3 times with DPBS and blocked with $10 \mathrm{mg} / \mathrm{ml} \mathrm{BSA}$ in DPBS for 2 hours at room temperature. The blocked plates were then washed with DPBS and incubated with recombinant Jagged1-Fc (R\&D Systems, 1277-JG) or CromPure IgG 
Fc (Jackson ImmunoResearch, 009-000-008) at a concentration of $2 \mu \mathrm{g} / \mathrm{ml}$ in $0.1 \%$ BSA and PBS for 4 hours at room temperature. After washing 3 times with DPBS, the cells were immediately seeded onto the coated plates in the appropriate medium, as described above for each cell type.

RNA isolation, RT-qPCR, and RNA-Seq. Total RNA was isolated from NICD2-ST2 cells or BMSCs with the RNeasy Mini Kit (QIAGEN, 74104). Reverse transcription was performed with 400 ng total RNA and iScript Reverse Transcription Supermix (Bio-Rad, 1708891). qPCR was performed with an ABI StepOne Plus machine in a 96-well format using SsoAdvanced Universal CYBR Green Supermix (BioRad, 1725274). Primer sequences are listed in Table 4.18S rRNA was used as an internal control, and relative expression was calculated by the $2^{-\Delta \Delta \mathrm{Ct}}$ method.

For RNA-Seq experiments, NICD2-ST2 cells were treated with vehicle or $500 \mathrm{ng} / \mathrm{ml}$ Dox for 24 hours before isolation of total RNA. Poly-A RNA was isolated from $20 \mu$ g total RNA using Oligo-dT beads [Dynabeads Oligo $(\mathrm{dT})_{25}$, Thermo Fisher Scientific] and used to generate the PCR-amplified cDNA library, which was then sequenced with the Illumina HiSeq 2000 as single reads extending 42 bases (Genome Technology Access Center [GTAC], Washington University School of Medicine). The raw data were demultiplexed and aligned to the reference genome ( $\mathrm{mm} 9$ ) using TopHat. The Partek Genomic Suite was used to assemble transcripts and analyze their expression. The RNA-Seq data were deposited in the NCBI's BioProject database (ID PRJNA488100).

Metabolite measurements. Subconfluent NICD2-ST2 cells growing in aMEM containing 10\% FBS were treated with vehicle or 500 $\mathrm{ng} / \mathrm{ml}$ Dox for 12, 24, or 48 hours. Glucose consumption and lactate production were measured with the Glucose (HK) Assay Kit (MilliporeSigma, GAHK20) and the L-lactate Assay Kit (Eton biosciences, 1200011002). ATP was measured in cell lysates with the CellTiterGlo Luminescent Cell Viability Assay Kit (Promega, G7573 ). For glucose uptake assays, the cells were incubated with $100 \mu \mathrm{M}$ 2-NBDG for 1 hour, and fluorescence intensity was measured according to the manufacturer's instructions (Glucose Uptake Cell-Based Assay Kit, Cayman Chemical, 600470).

Seahorse extracellular flux assays. NICD2-ST2 cells were plated at 5,000 cells/well in XF96 plates coated with Cell-Tak (BD Biosciences). On the next day, the cells were treated with $500 \mathrm{ng} / \mathrm{ml}$ Dox for 48 hours with or without inhibitors for the indicated durations before switching to the assay medium (Seahorse Bioscience, 101022-100, or MilliporeSigma, D5030-1L) and further incubation in a $\mathrm{CO}_{2}$-free incubator for 1 hour. Oligomycin (ATP synthase inhibitor), carbonyl cyanide p-trifluoromethoxyphenylhydrazone (FCCP; proton ionophore), antimycin A (complex III inhibitor), and rotenone (complex I inhibitor) (Seahorse XF Cell Mito Stress Test Kit, Seahorse Bioscience,
103015-100) were prepared in XF assay medium at final concentrations of $5 \mathrm{mM}$ for oligomycin and $1 \mathrm{mM}$ for the rest. At the end of the assays, protein concentrations were measured for normalization of the OCR and ECAR.

Measurement of mitochondrial superoxide and mitochondrial complex I activity. For mitochondrial superoxide measurements, NICD2ST2 cells were seeded in 96-well black wall tissue culture plates (Corning, 3904) and treated with vehicle or Dox for 6, 12, or 24 hours. The cells were then treated with the MitoSOX Red mitochondrial superoxide indicator (Molecular Probes, Thermo Fisher Scientific, M36008) at $5 \mu \mathrm{M}$ for 10 minutes at $37^{\circ} \mathrm{C}$, washed 3 times with DPBS, and measured at 510/580 nm with a plate reader (BioTek). The fluorescence intensity was normalized to the DNA content. Mitochondrial complex I activity was measured with the Complex I Enzyme Activity Microplate Assay Kit (Colorimetric) (Abcam, ab109721).

shRNA knockdown. Lentiviruses expressing shRNA were produced by transfecting HEK293T cells with pLKO.1 shRNA plasmids (Genome Center at Washington University) and the packaging plasmids pMD2.G and psPAX2 using Fugen6 transfection reagent (Promega). The viral media were added to the cells for 8 hours before being replaced with fresh growth media for an additional 48 hours. After treatment with Dox for 24 hours, the cells were harvested for RNA isolation and RT-qPCR. For glucose consumption and lactate production assays, the cells were harvested 48 hours after Dox treatment. shRNA targeting sequences are listed in Table 5.

Statistics. Statistical significance was calculated with either a 2-tailed Student's $t$ test or a 2-way ANOVA. A P value of less than 0.05 was considered significant.

Study approval. The studies in mice were reviewed and approved by the IACUC of Washington University.

\section{Author contributions}

SL conducted all experiments, prepared the figures, and wrote the Methods section. FL conceived the project and wrote the manuscript.

\section{Acknowledgments}

The work was partially supported by NIH grant R01AR055923 (to FL).

Address correspondence to: Fanxin Long, Children's Hospital of Philadelphia, 3651 Civic Center Blvd, ARC 902A, Philadelphia, Pennsylvania 19104, USA. Phone: 267.425.2071; Email: longf1@ email.chop.edu.

FL's present address is: The Children's Hospital of Philadelphia Research Institute, University of Pennsylvania, Philadelphia, Pennsylvania, USA.
1. Artavanis-Tsakonas S, Rand MD, Lake RJ. Notch signaling: cell fate control and signal integration in development. Science. 1999;284(5415):770-776.

2. Chiba S. Notch signaling in stem cell systems. Stem Cells. 2006;24(11):2437-2447.

3. Schroeter EH, Kisslinger JA, Kopan R. Notch-1 signalling requires ligand-induced proteolytic release of intracellular domain. Nature. 1998;393(6683):382-386.

4. Kopan R, Goate A. A common enzyme connects notch signaling and Alzheimer's disease. Genes
Dev. 2000;14(22):2799-2806.

5. Kopan R, Ilagan MX. The canonical Notch signaling pathway: unfolding the activation mechanism. Cell. 2009;137(2):216-233.

6. Kitagawa M. Notch signalling in the nucleus: roles of Mastermind-like (MAML) transcriptional coactivators. J Biochem. 2016;159(3):287-294.

7. Iso T, Kedes L, Hamamori Y. HES and HERP families: multiple effectors of the Notch signaling pathway. J Cell Physiol. 2003;194(3):237-255.

8. Hilton MJ, et al. Notch signaling maintains bone marrow mesenchymal progenitors by suppressing osteoblast differentiation. Nat Med. 2008;14(3):306-314.

9. Yorgan T, et al. Osteoblast-specific Notch2 inactivation causes increased trabecular bone mass at specific sites of the appendicular skeleton. Bone. 2016;87:136-146.

10. Lawal RA, et al. The Notch ligand Jagged1 regulates the osteoblastic lineage by maintaining the osteoprogenitor pool. J Bone Miner Res. 2017;32(6):1320-1331. 
11. Zanotti S, Smerdel-Ramoya A, Stadmeyer L, Durant D, Radtke F, Canalis E. Notch inhibits osteoblast differentiation and causes osteopenia. Endocrinology. 2008;149(8):3890-3899.

12. Engin F, et al. Dimorphic effects of Notch signaling in bone homeostasis. Nat Med. 2008;14(3):299-305.

13. Zhang H, et al. NOTCH inhibits osteoblast formation in inflammatory arthritis via noncanonical NF-кB. JClin Invest. 2014;124(7):3200-3214.

14. Garg V, et al. Mutations in NOTCH1 cause aortic valve disease. Nature. 2005;437(7056):270-274.

15. Mohamed SA, et al. Novel missense mutations (p.T596M and p.P1797H) in NOTCH1 in patients with bicuspid aortic valve. Biochem Biophys Res Commun. 2006;345(4):1460-1465.

16. Simpson MA, et al. Mutations in NOTCH2 cause Hajdu-Cheney syndrome, a disorder of severe and progressive bone loss. Nat Genet. 2011;43(4):303-305.

17. Isidor $\mathrm{B}$, et al. Truncating mutations in the last exon of NOTCH2 cause a rare skeletal disorder with osteoporosis. Nat Genet. 2011;43(4):306-308.

18. Canalis E, Schilling L, Yee SP, Lee SK, Zanotti S. Hajdu Cheney mouse mutants exhibit osteopenia, oncreased osteoclastogenesis, and bone resorption. J Biol Chem. 2016;291(4):1538-1551.

19. Zanotti S, et al. Sustained Notch2 signaling in osteoblasts, but not in osteoclasts, is linked to osteopenia in a mouse model of Hajdu-Cheney syndrome. JBiol Chem. 2017;292(29):12232-12244.

20. Esen E, Chen J, Karner CM, Okunade AL, Patterson BW, Long F. WNT-LRP5 signaling induces Warburg effect through mTORC2 activation during osteoblast differentiation. Cell Metab. 2013;17(5):745-755.

21. Karner CM, Esen E, Okunade AL, Patterson BW,
Long F. Increased glutamine catabolism mediates bone anabolism in response to WNT signaling. J Clin Invest. 2015;125(2):551-562.

22. Frey JL, et al. Wnt-Lrp5 signaling regulates fatty acid metabolism in the osteoblast. Mol Cell Biol. 2015;35(11):1979-1991.

23. Esen E, Lee SY, Wice BM, Long F. PTH promotes bone anabolism by stimulating aerobic glycolysis via IGF signaling. J Bone Miner Res. 2015;30(11):1959-1968.

24. Wei J, et al. Glucose uptake and Runx 2 synergize to orchestrate osteoblast differentiation and bone formation. Cell. 2015;161(7):1576-1591.

25. Landor SK, et al. Hypo- and hyperactivated Notch signaling induce a glycolytic switch through distinct mechanisms. Proc Natl Acad Sci US A. 2011;108(46):18814-18819.

26. Basak NP, Roy A, Banerjee S. Alteration of mitochondrial proteome due to activation of Notch1 signaling pathway. J Biol Chem. 2014;289(11):7320-7334.

27. Slaninova V, et al. Notch stimulates growth by direct regulation of genes involved in the control of glycolysis and the tricarboxylic acid cycle. Open Biol. 2016;6(2):150155.

28. De Bock K, et al. Role of PFKFB3-driven glycolysis in vessel sprouting. Cell. 2013;154(3):651-663.

29. Xu J, et al. NOTCH reprograms mitochondrial metabolism for proinflammatory macrophage activation. JClin Invest. 2015;125(4):1579-1590.

30. Fujimura S, Jiang Q, Kobayashi C, Nishinakamura R. Notch2 activation in the embryonic kidney depletes nephron progenitors. J Am Soc Nephrol. 2010;21(5):803-810.

31. Tu X, et al. Physiological notch signaling maintains bone homeostasis via RBPjk and Hey upstream of NFATc1. PLoS Genet. 2012;8(3):e1002577.

32. Clem B, et al. Small-molecule inhibition of 6-phosphofructo-2-kinase activity suppresses glycolytic flux and tumor growth. Mol Cancer Ther. 2008;7(1):110-120.

33. Maillard I, et al. Mastermind critically regulates Notch-mediated lymphoid cell fate decisions. Blood. 2004;104(6):1696-1702.

34. Hart PC, et al. MnSOD upregulation sustains the Warburg effect via mitochondrial ROS and AMPK-dependent signalling in cancer. Nat Commun. 2015;6:6053.

35. Ismaili N. Treatment trends and outcomes of smallcell carcinoma of the bladder: in regard to Koay et al. (Int J Radiat Oncol Biol Phys 2011 Oct 20). Int J Radiat Oncol Biol Phys. 2012;82(4):1319-1320.

36. Lee WC, Guntur AR, Long F, Rosen CJ. Energy metabolism of the osteoblast: implications for osteoporosis. Endocr Rev. 2017;38(3):255-266.

37. McCright B, Lozier J, Gridley T. Generation of new Notch2 mutant alleles. Genesis. 2006;44(1):29-33.

38. Logan M, Martin JF, Nagy A, Lobe C, Olson EN, Tabin CJ. Expression of Cre Recombinase in the developing mouse limb bud driven by a Prxl enhancer. Genesis. 2002;33(2):77-80.

39. Ong CT, et al. Target selectivity of vertebrate notch proteins. Collaboration between discrete domains and CSL-binding site architecture determines activation probability. J Biol Chem. 2006;281(8):5106-5119.

40. Megason SG, McMahon AP. A mitogen gradient of dorsal midline Wnts organizes growth in the CNS. Development. 2002;129(9):2087-2098.

41. Weng AP, et al. Growth suppression of pre-T acute lymphoblastic leukemia cells by inhibition of notch signaling. Mol Cell Biol. 2003;23(2):655-664. 